\title{
Impact of Demographics on Healthcare Utilization
}

\author{
Andrew Walsh* \\ Health Monitoring Systems, Inc, Pittsburgh, PA, USA
}

\section{Objective}

To determine if data collected for syndromic surveillance can inform policy questions related to emergency department utilization and inpatient readmission

\section{Introduction}

The Affordable Care Act (ACA) was promoted with two goals: expanding health insurance coverage and reducing healthcare costs. Expanded coverage is expected to partially reduce costs. Emergency department (ED) visits are costlier than comparable primary care physician visits. If uninsured patients use the local ED more often than insured patients with comparable conditions, insuring them may change usage and lower costs.

Some reports in the literature do not fit this model of ED usage. In one study, nonurgent ED visits were mainly the result of patient uncertainty about the severity of their condition. While trained medical personnel distinguished urgent and nonurgent cases after the fact, initial presentations were similar. In Oregon, an expansion of Medicaid increased health insurance coverage; ED usage increased rather than decreased. Thus, the motivating narrative about insurance coverage and ED usage informing the ACA may not be the complete story.

Reduction of hospital readmissions is also expected to cut costs under the ACA. Hospital process improvements are expected to realize this reduction. Recently it was reported that up to $60 \%$ of hospital readmissions are predicted by patient demographics, raising questions about how much control a hospital has over its readmission rate. This research will examine whether data collected via syndromic surveillance can corroborate these findings.

\section{Methods}

US census data was obtained for New Jersey $(\mathrm{NJ})$, Ohio $(\mathrm{OH})$, and Pennsylvania (PA) and aggregated to zip code tabulation area (ZCTA) for comparison with hospital data which was only available at that granularity.

ED registration counts were obtained from 312 hospitals in NJ (48), OH (167), and PA (97) for 2011-2013. ED usage rates for the ZCTAs were calculated using the maximum of total population values for that ZCTA and adjusted for days reporting. Readmission rates were obtained from a subset of these hospitals which send inpatient data via their syndromic surveillance feed.

Regression analysis used R v3.1.1, with packages lme4 v1.1-7 and MASS v7.3-33.

\section{Results}

ED registrations were tabulated for 3,537 ZCTAs in NJ, OH, and PA. A total of 34,412,761 registrations were observed for a usage rate of 0.337 registrations per person per year.

Figure 1 shows the percentage of the ZCTA population with public insurance coverage as an indicator of ED usage. ZCTAs were assigned to quartile groups based on public insurance coverage rate. The more people in a ZCTA with public insurance, the more ED visits. Regression confirmed these differences as statistically significant. The best fitting multivariate regression model of ED visits included several insurance covariates. Demographic data accounted for $59 \%$ of ED usage variation between ZCTAs.
Inpatient admissions and readmissions for 9 months in 2013 and 2014 were tabulated for each ZCTA in NJ, OH, and PA; 1,736 ZCTAs were represented in the dataset. A total of 425,291 admissions and 60,501 readmissions were observed for a readmission rate of 0.142 readmissions per admission.

Regression models were also created for readmission rates. Several insurance covariates were also determined to be significant for these models. Demographic data accounted for $53 \%$ of the variation in readmission rates between hospitals.

\section{Conclusions}

The consistent association between insurance coverage within a ZCTA and healthcare utilization of residents from that ZCTA warrants further investigation. Increasing coverage may not have the expected effect on healthcare usage and costs. The influence of demographic factors also indicates that hospitals may have limited ability to improve the targeted outcomes.

As with any study of aggregate data, there is the possibility of conflating group observations with individual effects. Before implementing policy changes, it is necessary to replicate these correlations at an individual level.

Fig 1: ED Usage by Public Insurance Coverage Rate

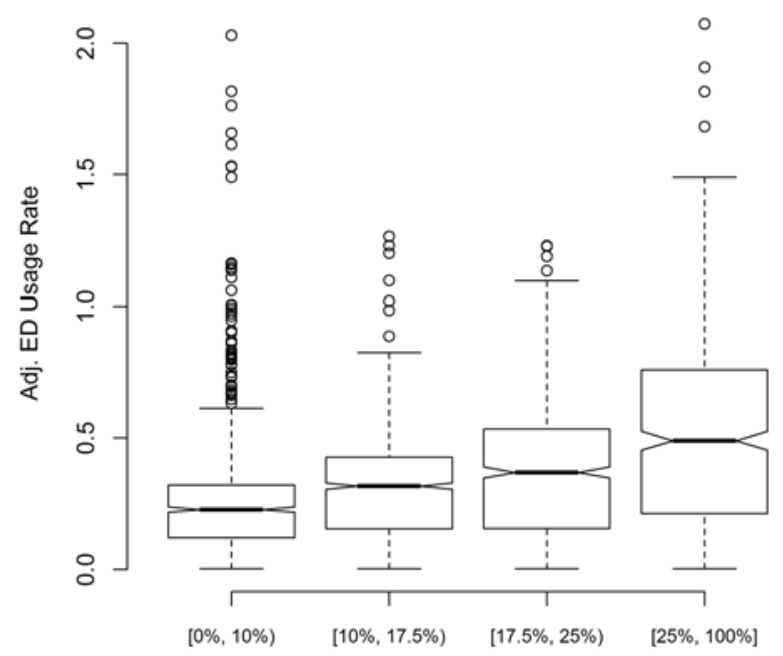

Keywords

Affordable Care Act; Census Data; Readmission; Medicaid

\section{Acknowledgments}

We thank the New Jersey, Ohio, and Pennsylvania Deparments of Health for funding support and data for this work.

\section{*Andrew Walsh}

E-mail: andy.walsh@hmsinc.com 